\title{
Energy returns and the long-run growth of global industrial society
}

\author{
Andrew Jarvis, Lancaster Environment Centre, Lancaster University \\ a.jarvis@lancs.ac.uk
}

\begin{abstract}
The extreme interconnectedness of energy and economic systems will tend to confound any attempt to estimate the energy return on investment at anything other than the global scale. Here, I apply a very simple model of global energy use to specify the dynamic characteristics of global-scale Energy Returns On Investment $\left(\mathrm{EROI}_{\mathrm{G}}\right)$. This suggests that the observed long-run relative growth rate of $\sim 2.5 \% \mathrm{yr}^{-1}$ in global primary energy use is associated with an equilibrium return from infrastructure investments of $2: 1$, with returns accruing with a time constant of 40 years. The analysis also attempts to show how growth leads to reductions in the supply efficiency of energy, and how this decline is offset by increases in the efficiency with which industrial society can extract useful work from primary energy flows. This observed preservation of the overall energy efficiency of the global energy system implicates variations in the decay/decommissioning rate of infrastructure in observed 'long-wave' like variations in the relative growth rate of global primary energy use, and hence $\mathrm{EROI}_{\mathrm{G}}$.
\end{abstract}

\subsection{Introduction}

Because of the way economies co-evolve with their use of energy, it is hard to be precise about the nature of the dependencies between the two (Stern, 2010). However, in standard economic frameworks, energy use is generally assigned around $5 \%$ of productivity in line with observed short-term production costs (Dennison, 1979; Ayres and Warr, 2010; Kummel, 2011). This appears to contrast with everyday experience, which suggests that, like food, energy use is central to all socioeconomic activity, and if energy flows were withdrawn, global economic output would cease entirely rather than simply falling by $5 \%$ (Ayres and Nair, 1984). The centrality of energy use to the global economy is also underscored by the fact that many of the largest manmade infrastructures on the planet are for energy acquisition, distribution and use, and likewise many of the largest companies in the world (http://fortune.com/global500/list/).

Observations such as these, along with the growing importance of understanding drivers of energy use and resultant greenhouse gas emissions, have encouraged a growing number of researchers to favour placing energy at the heart of their analysis of industrial society, rather than it being a somewhat peripheral factor of production. Such approaches naturally lean toward either thermodynamic (e.g. Garrett, 2011), or metabolic (e.g. Cleveland et al., 1984) frameworks, because of the explicit and central framing around energy flows, and their role in maintaining and developing the form and function of systems and processes. Although some have attempted to integrate both economic and biophysical perspectives (e.g. Ayres and Warr, 2010; Kummel, 2011), there tends to be a tension between the two approaches, 
given that one emphasises the primacy of people in determining system behaviour, whereas the other emphasises the primacy of biophysical regularities, such as the first and second laws of thermodynamics.

Energy Return On Investment (EROI) has been a valuable concept in helping bridge these economic and biophysical perspectives by articulating the potential viability of energy sources in relation to their yield of net available energy to industrial society (Cleveland et al., 1984; Hall et al., 1986). However, despite its conceptual elegance, EROI has proved particularly difficult to define and measure because this requires the demarcation of process/system boundaries. As with any such process-based analysis, the complexity of the wider economic system(s), and in particular the nature of the interlinkages between components, makes the definition of clear internal boundaries problematic at best.

Rather than considering the energy returns of individual energy sources as is commonly done, one way of simplifying matters could be to take the entire global energy system and its associated portfolio as a whole. At the global aggregate scale, the system boundaries that define energy returns are potentially far simpler because one generally avoids the need to identify internal system boundaries, whilst the global boundary is comparatively well defined by the flow of primary energy from the environment into industrial society. This is also the scale at which the critical impacts of climate change and the required transitions in the global energy portfolio are framed and, as a result, such an analysis might bring some muchneeded clarity to an otherwise notoriously complicated but important space. Therefore, although one may sacrifice detail on specific processes at this scale, and some of the derived states may become somewhat abstracted from everyday micro-experience, removing internal boundary ambiguities may provide a clearer picture of the systemic drivers of global energy use.

In this paper, I develop a global-scale analysis of energy returns based on recent work by Jarvis et al., (2015). In that work, they present a very simple endogenous growth model that attempts to account for two important observed features of the historic pattern of global primary energy use (GPE): a. that the long-run relative growth rate has been somewhat conserved at $\sim 2.5 \% \mathrm{yr}^{-1}$; and $\mathrm{b}$. that the fraction of this energy that is used to acquire and relocate resources has increased as the system has grown. These observations have several important implications for energy return analysis. First, overall rates of return on energy use must be also be somewhat conserved at the global scale if the relative growth rate of primary energy use is similarly conserved. Secondly, any observed historic declines in specific energy return measures such as EROI (e.g. Murphy and Hall, 2011), may be related to growth-induced increases in resource supply costs, but these declines must be offset by increases in the efficiency of energy use elsewhere in the system, in order to produce a somewhat stationary long-run relative growth rate. Finally, a process experiencing $\sim 2.5 \% \mathrm{yr}^{-1}$ growth has a dynamic timescale of $0.025^{-1}=40$ years and, as a result, energy investments on average yield returns over such timescales. Therefore, measures such as EROI need to consider the dynamics operating over these timescales (Dale et al., 2012a). The aim of this paper is to illustrate these three points and relate them to our understanding and management of the global energy system. 
The paper is organised as follows: Section 2 sets out the global-scale endogenous growth model. Section 3 then uses this model to develop a dynamic definition of global EROI, $\mathrm{EROI}_{\mathrm{G}}$. Section 4 explores how growth-induced increases in resource supply costs lead to declining energy returns, but also how these might be balanced by adjustments in efficiency elsewhere in the system such that overall returns and hence growth is maintained. Section 5 explores systematic variations in observed growth rates of GPE about their long-run value, and Section 6 offers some conclusions.

\subsection{Modelling framework}

There are numerous global energy system models (see Jebaraj and Iniyan, 2006; Bhattacharyya and Timilsina 2010 for reviews) that vary in complexity depending on application. Here I present possibly one of the simplest in that it makes no attempt to resolve any detail beyond what is necessary to describe the observations that a. GPE use has, on average, grown at $\sim 2.5 \% \mathrm{yr}^{-1}$ for at least the last century, and $\mathrm{b}$. the efficiency of supplying resources to points of final consumption has fallen consistently as the system has grown (see below). In this framework, energy use is assumed to underpin all economic activity such that the real economy and the global energy system represent one and the same thing over the timescales being considered.

\section{Components of primary energy use}

GPE is the total annual energy consumption (or annual average power demand) of industrial society made up from wood, fossil fuels, renewables, nuclear and food. Estimates of this for 1900 to 2014 compiled by De Stercke (2014) are shown in Figure 1i, and currently (in 2014) this is around $600 \mathrm{EJ} \mathrm{yr}^{-1}$ or $19 \mathrm{TW}$. Let us denote GPE, $x$. Although this energy flow is used for a vast array of differing activities, here I start by considering it as being comprised of just two components: the energy used to acquire resources from the environment and to distribute them within industrial society (or the supply energy use, $x_{S}$ ); and the residual that is net available to do things beyond resource acquisition and distribution (or net available energy use, $x_{A}$ ).

$$
x=x_{S}+x_{A}
$$

Defining the partitioning of primary energy use outlined in equation (1) is nontrivial. This is because energy used for resource supply includes both the running cost (which see instantaneous returns), and investments in the associated infrastructure (which see returns over the investment timescale). The former are commonly accounted for in annual energy input-output statistics such as those reported by the International Energy Agency (IEA). These are associated with the operation of infrastructure for the extraction and relocation of resources from where they are in the environment, to where they are needed within industrial society. Although there are considerable uncertainties associated with the specification of these costs (for example, points of end-use are invariably treated as points of 
sale for convenience), it is the uncertainties surrounding the embedded energy invested in the associated infrastructure that present the biggest challenge (Dale et al., 2012a). The reason for this is because there are no clear system boundaries for these investments because a. the associated supply chains are infinite, b. this stock of infrastructure is the product of all prior investments, and c. all infrastructure is networked together. As a result, defining the infrastructure investment component of $x_{S}$ encounters the same truncation error problems that plague all process-based life-cycle analyses when one is analysing subcomponents of global systems (Hendrickson et al., 1998; Lenzen, 2001).

In addition to taking a global perspective, one way to overcome the difficulty associated with specifying infrastructure in equation (1) is to re-define $x_{S}$ such that it only accounts for running resource supply processes, with some or all of $x_{A}$ being used for investing in infrastructure. This marks an important departure from standard practice in EROI analysis which invariably attempts to include running costs alongside infrastructure investments. In my framework, running costs are simply committed expenditure to operate existing infrastructure, whereas investments represent choices over what infrastructure to create using net available energy. This approach provides an unambiguous physical demarcation in the system because investments are associated exclusively with the transition of energy flows into infrastructural stocks at points of 'end use.' Put another way, energy is used to either move mass, or make infrastructure, with only the latter constituting investment. Furthermore, infrastructure is, therefore, defined by the embodiment of energy in specific configurations of resource-derived materials, and the function of this infrastructure is to facilitate access to future resource flows (Garrett, 2012).

Before seeing how investments of net available energy lead to the accumulation of infrastructure, it is important to acknowledge that, although we have separated processes of acquisition/distribution and infrastructure fabrication here to reflect the difference between running costs and investments in infrastructure, these processes occur intermittently throughout the system. As such, they are very difficult to separate spatially. For example, the energy used to do the work of digging coal from the ground and relocate it to a steel plant is clearly an acquisition/distribution flow. That coal can then be invested to smelt ore to produce a stock of steel, which is part of an infrastructure forming process. However, that steel can then be relocated back through a supply chain to make a bucket wheel excavator for harvesting more coal, with the making of the excavator parts from the steel requiring yet more investment of net available energy, and the relocation of these steel parts and their assembly requiring yet more supply energy use. Interestingly, the flow of coal from the mine is the flow of energy and its mass carrier (largely carbon). However, the movement of the steel is also a flow of energy and mass, and we refer to that energy as the embodied energy in the steel. As a result, the networks moving resources through industrial society are always moving mixtures of both energy and mass (Jarvis et al., 2015).

Dale et al., (2012b) also differentiate explicitly between capital investments and running costs when modelling the EROI of the global energy system. They specify infrastructure associated with energy acquisition and distribution, and that which is associated with other functions performed by industrial society. Although an appealing and obvious choice to make, and the prevailing view of energy systems, this approach raises a critical issue: is it correct to differentiate between energy producing infrastructure, such as an oil rig, and what 
is perceived to be non-energy producing (and hence by implication 'useful') infrastructure, such as a house? In an energy system sense, it can be argued that the house is as necessary for acquiring and distributing energy resources as the oil rig, because it houses those servicing and operating the system, either directly or indirectly. Similarly, the oil rig is as integrated into the fabric of industrial society as the house, if we believe there is such a thing as a global energy system, where all components are connected by acquisition/distribution networks. Furthermore, both the house and the oil rig are constructed from the fraction of $x$ that is not used to either acquire or distribute resources, so the addition of either a house or an oil rig to industrial society must be considered to be material growth of the system. Therefore, although we might believe the house is somehow more 'useful,' and hence more closely linked to net available energy flows, this distinction is possibly an illusion in terms of the global energy system, given the highly interconnected nature of infrastructure and its ubiquitous use of energy.

There is a direct parallel here with biological systems such as the body. The fabric of the human body includes all distribution infrastructure (mouth, throat, intestine, bronchi, arteries, veins, lymph system, etc.), and the growth of the body includes material additions to, or investments in these networks, as much as it does to the soft and hard tissues these networks service with resources. Furthermore, these soft and hard tissues support the operation of the acquisition/distribution systems, as the acquisition/distribution systems support the operation of these tissues. Together, they form an integrated whole that we call the body, in the same way that acquisition/distribution systems are part of the integrated whole of industrial society. Therefore, differentiating between elements of either the body or industrial society based on 'usefulness' is largely arbitrary. As a result, I adopt the possibly surprising position that, at the global scale, $x_{A}$ is used exclusively for investing in infrastructure, and that we make no distinction between energy and non-energy infrastructure: all infrastructure is used for energy acquisition and distribution either directly or indirectly (Garrett, 2012). Therefore, $x_{S}$ is used exclusively to run infrastructure and hence is not an investment per se.

\section{Growth in energy use}

In addition to GPE, Figure $1 \mathrm{i}$ also shows an estimate of Gross World Product (GWP) 1900 to 2014, again taken from De Stercke (2014). Although there is significant variability about the trend (see Section 5), by far the dominant feature of these data, explaining more than $95 \%$ of the variance (see Table 1), is the underlying tendency for growth to be exponential in both GWP and GPE. Using these data, I estimate that GPE expresses a long-run relative growth rate of $2.7( \pm 0.04) \% \mathrm{yr}^{-1}$ and GWP $3.1( \pm 0.04) \% \mathrm{yr}^{-1}$ (Table 1) suggesting they share a common exponential scaling relationship (Figure $1 \mathrm{i}$ inset). Using a slightly different compilation of GPE based on the Grübler (2008) and British Petroleum (2011) data, Jarvis et al., $(2012 ; 2015)$ estimated that the 1850 to 2010 relative growth rate of GPE was 2.4 ( \pm $0.04) \% \mathrm{yr}^{-1}$. As a result, it appears there is some sensitivity of this estimate to the data length and sources used. As a result, hereon I will assume a rounded value for the long run relative growth rate of GPE of $2.5 \% \mathrm{yr}^{-1}$ to avoid over-reporting the precision with which we know this number. 
The analysis hereon focuses largely on the first order exponentiality of GPE. Not only is this the overriding feature of the evolution of these data, but growth is the central economic calculus of the current global socioeconomic system, and it is hard to envisage this altering for the foreseeable future without some form of radical, systemic change, precipitated by either foresight, or physical limits. Moreover, it appears that this central calculus is not simply growth, but the appearance of preferred growth rates, with periods above this considered unwanted inflationary episodes, and periods below considered unwanted recessionary episodes. For reference, Figure $1 \mathrm{iii}$ shows an estimate of these underlying variations in the relative growth rate in GPE which give a strong impression of there being a 'long-wave' in this measure of the global economy (see Section 5; see also Jarvis and Hewitt, 2014; Manoli et al., 2016).

A relative growth rate of $r=2.5 \% \mathrm{yr}^{-1}$ corresponds to a growth timescale of $r^{-1}=40 \mathrm{yrs}$. Jarvis et al., (2015) offer an argument based on working lifetimes as to why growth of this kind is preferred in the real economy. Briefly, growth rates of this order can be seen as optimal if you are attempting to maximise returns over your working lifetime, in order to secure prospects in retirement (Jarvis et al., 2015). As we will discuss later, this highlights why pensions are an important investment instrument for securing long-run growth. We also now have a definition of long-run, i.e. behaviour expressed on this $\sim 40$-year timescale. Not only is this justified because of the dominance of exponentiality in the data in Figure 1, from a climate change perspective, the climate system receiving the associated anthropogenic greenhouse gas emissions is, in effect, a low-pass filter, responding only to the long-run dynamics of industrial society. As a result, we rightly focus on these dynamics above all else when considering avoiding dangerous climate change. Furthermore, only a modest lag is required in any socioeconomic feedback regulating growth at $\sim 2.5 \% \mathrm{yr}^{-1}$ to produce the type of variations seen in Figure 1iii.

Following Garrett (2011), the energy-related growth in the real economy is ultimately determined by the feedback between investments of energy in the material fabric of society (which hereon we refer to as 'infrastructure'), $X$, and the use of this infrastructure in the acquisition of primary energy flows, $x$. Infrastructure can be thought of as the embodiment of energy investments within materials (Garrett, 2012), and hence the conservation of these energy investments is given by

$$
\frac{d X}{d t}=\eta x-k X
$$

where $\eta$ is the fraction of the primary energy flow $x$ that drives infrastructure creation, and $k$ is the average decay rate of that infrastructure.

The average lifetime of large energy infrastructure appears to be $\sim 40$ years (Davis et al., 2010), giving a turnover rate of $40^{-1}=2.5 \% \mathrm{yr}^{-1}$ for this stock. This is also the average timescale that technologies appear to diffuse into the global economy (Grübler et al., 1999) and hence, by implication, leave it. Therefore, we might conclude that the turnover of all infrastructure is somehow regulated by the turnover of large energy infrastructure which provides the skeleton of the real economy. Because this is also the long-run relative growth 
rate of $x$, it appears this growth rate is mirrored in the turnover rate of infrastructure, a situation that would be optimal if innovation and the introduction of new infrastructure were required to maintain real growth at this rate (Jarvis et al., 2015). From these observations, I infer $k \approx r$ on average across the global infrastructure portfolio. This of course masks the fact that there is considerable variation about this figure, with some infrastructure turning over much more rapidly (e.g. a paper cup) and some much less so (e.g. a road or rail cutting).

Given equation (2), for $x$ to be long-run exponential, as shown in Figure 1, there must be linear scaling between the stock $X$ and the flow $x$ (Jarvis et al., 2015) i.e.

$$
x=\frac{X}{\lambda}
$$

where $\lambda$ is the effective residence time of the energy flowing through the infrastructure of industrial society. We are now able to describe the endogenous real growth of industrial society in energy terms as,

$$
\frac{d X}{d t}=(\eta / \lambda-k) X=r X
$$

with respect to infrastructure or,

$$
\frac{d x}{d t}=(\eta / \lambda-k) x=r x
$$

with respect to primary energy use.

If $k=r$, then to get a relative growth rate of $2.5 \% \mathrm{yr}^{-1}$ in both $X$ and $x$, annual additions to infrastructure must be twice the decay rate. Therefore, from equation (4b), $\eta / \lambda=2 k=2 r$. By definition, annual energy flows are fully consumed within the year and hence have an effective residence time of $\lambda=1 \mathrm{yr}$. If so then $\eta=0.05$ i.e. overall, in the long-run, industrial society is $5 \%$ efficient at translating the annual primary energy flow into material additions of infrastructure. Ayres (1989) estimated that the US energy system was $2.5 \%$ efficient when translating primary energy flows into material growth whilst Kummel (2011) estimates this to be closer to $10 \%$. If $5 \%$ of $x$ is translated into infrastructure creation, the remaining $~ 95 \%$ must be committed to the annual running costs of the real economy and waste.

\section{Global energy use efficiencies}

$\eta$ is the dimensionless efficiency with which primary energy use drives infrastructure creation and can be thought of as the product of a series of efficiencies describing how energy flows from the environment to points of final use where infrastructure is created. Here I consider the following fundamental components of this aggregate efficiency as being, 


$$
\eta=\eta_{U} \eta_{S} \eta_{M}
$$

where $\eta_{U}$ is the efficiency of the primary energy portfolio at doing useful work, $\eta_{S}$ is the efficiency of the supply networks linking environmental energy sources to points of end use (which in this case are the points of manufacture of $X$ ), and $\eta_{M}$ is the efficiency of the final embodiment of end-use energy into physical infrastructure through manufacturing.

In addition to GPE data, Figure $1 \mathrm{i}$ also shows estimates of both 'final' and 'useful' energy 1900 - 2014 taken from the De Stercke (2014) database. The difference between the primary and useful energy measures the proportion of primary energy able to do work throughout the system (Grübler, 2012). The difference between primary and final energy is the energy used to relocate energy and its mass carriers from the environment to where it is needed. These data are derived from the IEA final energy estimates where final is defined as point of sale for convenience. However, this treats all transport as a final use when, clearly, transport is the movement of embodied energy and its mass carriers, including people. As a result, here I have also removed all transport from the final energy data in the De Stercke (2014) database. Jarvis, et al. (2015) also remove all energy used in mining, quarrying, fishing and agriculture given these activities are also associated with relocating energy and mass carriers from the environment to points of final consumption. Unfortunately, the De Stercke (2014) database does not have these sub-categories of energy use and, therefore, the final energy data presented in Figure 1 will be an over-estimate relative to those compiled by Jarvis, et al. (2015).

I take the final energy data shown in Figure $1 \mathrm{i}$ to be the energy that is net available to create infrastructure, $x_{A}$. Figure $1 \mathrm{ii}$ also shows the ratio of final to primary energy flow, $x_{A} / x$, for the period 1900 to 2014 using the De Stercke (2014) data. I take this ratio to be the efficiency of the supply system delivering energy (including embodied energy) to points of end use, $\eta_{S}$. This is currently $\sim 0.5$ and has fallen throughout the observation period (see Figure 1ii, Section 4 and also Jarvis et al., 2015 for extensive discussion of this). To reiterate, included in this estimate of supply efficiency are all energetic losses that occur between environmental source and the sinks at points of end-use where infrastructure is manufactured. Also presented in Figure $1 \mathrm{ii}$ is an estimate of the usefulness efficiency, $\eta_{U}$. Because De Sterke (2014) specifies useful energy flow downstream of the final energy flow, this estimate has been taken as the average of the ratios of useful to primary and useful to final energy flows hence assuming usefulness remains unchanged as flows traverse the energy supply system.. I estimate $\eta_{M}=\eta / \eta_{U} / \eta_{S}$ because I am assuming $\lambda=1 \mathrm{yr}$ and $\eta=$ 0.05 (see Section 4 also).

In summary, the relative growth rate of the infrastructure of industrial society (or its annual primary energy use) is determined by four factors in this analysis: the residence time of annual energy flows $(\lambda)$; the efficiency with which primary energy can do useful work $\left(\eta_{U}\right)$; the efficiency with which energy is supplied to points of final use $\left(\eta_{S}\right)$; the efficiency with which this end-use energy flow is translated into infrastructure additions $\left(\eta_{M}\right)$; and the decay rate of that infrastructure $(k)$. These factors determine energy returns and return rates in this framework. A full systems block diagram for this framework is given in Figure 2. 


\subsection{Global scale dynamics of energy returns}

In its simplest possible form, EROI can be expressed as the ratio of energy returned to energy invested i.e.,

$$
\mathrm{EROI}=\frac{\text { energy returned }}{\text { energy invested }}
$$

In economics, returns are generally calculated using the net yield, whereas EROI analyses have used both net and gross energy yields depending on the system being studied (Brandt et al., 2013; King et. al., 2014). Here I will define EROI in terms of gross yield returned, because at the global scale, this is the primary energy flow, $x$.

Unfortunately, in a system undergoing exponential growth, the energy flows in any particular year are a combination of the effects of all prior infrastructure investments (Garrett, 2014). However, we can overcome this complication by considering equation (4b) in the following annually sampled form

$$
x(t)=(1-k) x(t-1)+(\eta / \lambda) x(t-1)
$$

Expressed in this way we have deliberately partitioned the endogenous growth dynamics driving the exponential increases in GPE (as detailed in equation (4b)) into annual energy inputs used to create infrastructure (the last term on the right-hand side of equation (7)), and the subsequent internal cycling and dissipation of that embodied energy in the form of infrastructure decay (the first term on the right-hand side of equation (7)). In structuring equation (4b) in this way, we have broken the feedback between $x(t)$ and $x(t-1)$ that ultimately gives rise to the exponentiality of the system. Therefore, we can now explore how an investment input at $t$ - 1 gives rise to future output returns, without the confounding effects of the implied feedback between $x(t)$ and $x(t-1)$.

The partitioning of the dynamics in equation (4b) into an input and an output of primary energy becomes clearer if we form the appropriate input-output relationship, or transfer function, which describes how energy investments in any one year translate into returns in the following year(s). Using the backward shift operator $\mathrm{z}^{-1}$ where $\mathrm{z}^{-1} x(t)=x(t-1)$, equation (7) can be written as the following annually sampled input-output relationship,

$$
x(t)=\frac{\eta / \lambda}{1-(1-k) \mathrm{z}^{-1}} \cdot x(t-1)
$$

This transfer function is exactly what we want when defining global energy returns because it defines how the investment of energy in one year translates into energy returns in following years when isolated from the effects of exponential growth. 
From equation (8) the first thing to note is that all the primary energy flow input is used in one year to drive the primary energy flow outputs in the following years. This suggests from the outset that all energy use at the global scale is spoken for, and the concept of 'net available' energy flows that can be freely allocated within industrial society is an illusion. This is not to say that we do not find this energy use important, as clearly we do. But to consider a portion of it as available is to consider the things it would be used on as being somehow separated from energy acquisition/distribution/end-use activities, including the growth of these activities resulting from the creation of infrastructure. As argued for above, perhaps this is not the right way to see the use of energy, and it would be better to conceive of this as a genuinely integrated global system. In this context, what we value is not our ability to freely spend a residual of net available energy flows, but rather the entire use of the primary energy flow and its growth.

In line with the discussion above, equation (8) also shows that $\eta / \lambda$, or $5 \% \mathrm{yr}^{-1}$, of the primary energy flow is translated directly into energy flows in the following year through the direct effect of an annual investment in infrastructure (see Figure 3). If one viewed this as the infrastructure investment for acquiring energy flows and we observe an output of $x(t)$ in the following year, we would conclude that the system has a short-run (annual) EROI of $0.05^{-1}=$ 20:1. Because this EROI estimate relates solely to short-run energy flows, it is akin to a power ratio (Herendeen, 2015). It is also close to average value of EROI quoted for conventional energy sources (Dale et al., 2012a). Standard EROI estimates do include running costs, do not include wider investments in the infrastructure, and do attempt to include all subsequent returns. Therefore, that the short-run EROI of the global energy system appears to mirror the long-run EROI of specific conventional energy sources is surprising. This suggests an equivalence between the proportion of energy invested in producing and running wider infrastructure that standard EROI estimates typically exclude, and the future energy returns from specific energy sources that they attempt to include.

Clearly the short-run estimate of global EROI of 20:1 discussed above ignores the fact that the residual $95 \%$ of primary energy use is also required to run this global system and, more importantly, that all subsequent returns of energy in following years need to be considered as identified in equation (6). In terms of equation (8), this is equivalent to the summation of all outputs of primary energy resulting from a unit input of primary energy at time $t$. The annual outputs of primary energy for a unit input is equivalent to the unit impulse response of equation (8). The reader can readily compute this for themselves using equation (7) by replacing the last term on the right hand side with $(\eta / \lambda)(1)$ for $t=0$ and $(\eta / \lambda)(0)$ thereafter. For this the initial condition is $x(0)=0, \eta / \lambda=0.05 \mathrm{yr}^{-1}$ and $k=0.025 \mathrm{yr}^{-1}$. This is shown in Figure 3. In year $t=1 x(t)$ is $5 \%$ of the unit input reflecting the first year of output from the infrastructure investment. In subsequent years this decays at $2.5 \% \mathrm{yr}^{-1}$ as this infrastructure is slowly eroded by decay.

The total output from the unit of investment is the sum of all annual outputs over time, which is also the EROI response over time, given that we have made a total input investment of one unit of primary energy at $t=0$. This EROI rises from 0.05 in the first year, breaks even $(\mathrm{EROI}=1)$ after $\ln (2) / k=28$ years, and rises to $\eta / \lambda / k=2$ in equilibrium with a time constant of $k^{-1}=40$ years. Therefore, at the global scale and over the full lifetime of any energy input, 
the EROI of the global energy system is $2: 1$ (Figure 3). Hereon I refer to this as the global $\mathrm{EROI}\left(\mathrm{EROI}_{\mathrm{G}}=\eta / \lambda / k\right)$.

$\mathrm{EROI}_{\mathrm{G}}$ parallels what has been previously referred to as 'societal' EROI (Hall et al., 2014), albeit at the global scale, in that it attempts to capture returns in relation to all energy uses, rather than those simply associated with a specific energy supply sub-system such as that for oil. Although an $\mathrm{EROI}_{\mathrm{G}}$ of 2:1 seems low given estimates of specific energy supply subsystems are up to an order of magnitude larger, it is still greater than one, reflecting a global system experiencing growth. What is probably more surprising for those familiar with EROI analyses is that an investment of primary energy only breaks even after just short of 30 years. What this reflects is that current flows of primary energy are much more dependent on prior investments in infrastructure and the energy used to run it than present investments. As can be seen from Figure 3, investments made 40 years ago still contribute around one third $\left(1-e^{-1}\right)$ of present-day energy outputs, and those made 100 years ago still some $10 \%$. Qualitatively this is what we observe, where significant portions of industrial society that were constructed decades ago are still actively contributing to both the supply and demand for energy.

It is interesting to note that solar photovoltaic systems appear to have recently approached grid parity with conventional electricity production and delivery systems in certain locations (Louwen et al., 2016) and this appears to have coincided with these systems now achieving 30 -year design lifetimes. This is in line with the result in Figure 3 which shows EROI breakeven occurs at $\ln (2) / k=28$ years at the global scale and hence any shorter design lifetime represents a technology unable to yield net returns to wider society. In contrast, somewhat surprisingly the EROI of solar photovoltaic systems appears to have remained somewhat static despite the significant improvements in efficiency and reductions in unit costs experienced over the last 30 years (Dale et al., 2012b). One would predict therefore that only when solar photovoltaic system design lifetimes reach 40 years, as appears to be the norm for large energy infrastructure (Davis et al., 2010), can it be considered a mature technology that will be ubiquitously invested in, hence becoming 'conventional.'

\subsection{The historic decline of global energy returns}

Much of industrial society was founded on energy dense fossil fuel sources, which provided large enough energy yields to overcome the relatively low efficiency, at that time, of converting these energy resources into useful work (Hall et al., 1986). However, it appears from some measures that the returns on these sources have since fallen steadily (Guilford et al., 2011). The reason often given for this is that society is exhausting the more easily acquired energy resources and hence has had to look to energetically less attractive resources (Guilford et al., 2011; Hall et al., 2014). There are two possible reasons for returns to fall in this context: First, the energy density of a source-type will decline over time as we consume the higher-quality, more energy-dense reserves first. Under these conditions progressively more energy is required to concentrate less-dense sources to useful levels. Secondly, we tend to exploit resources nearer to points of end-use first, before turning to resources further away. Under these conditions progressively more energy is required to relocate energy and its mass carrier in space to where it is needed. 
Although at first sight these two processes appear different, they can largely be seen as having the same effect on supply efficiency because in essence both processes involve relocating energy and its mass carrier in space. Furthermore, we can (and do) substitute one for the other when deciding which energy resource to exploit. Because of this, I argue one can explore observed declines in energy returns largely from the perspective of the effects of increases in the 'size' of industrial society on supply losses and hence declines in the supply efficiency of energy resources. As industrial society grows, environmental resources effectively become further away from points of end-use and hence the networks involved in the supply of these resources must be extended (Jarvis et al., 2015). This is not a linear process because as more finely divided resources are having to be distributed to ever more people, not only does the overall distance between the two increase, there are also disproportionate increases in energy loss in the terminal links between the two. This is known as the last mile problem in distribution networks (Rodrigue et al., 2009), and applies as much to the first mile as it does the last when considering environmentally-derived resources.

Providing the average energy density of infrastructure is somewhat conserved (Garrett, 2012), the space being occupied by industrial society increases proportionally with $X$. Jarvis et al., (2015) argue this increase in the physical size of industrial society requires extensions to existing acquisition/distribution networks, and that these extensions lead to increased energy loses due to more work having to be expended to move mass through these networks. Indeed, this can be seen as the primary motivation driving innovation on distribution technologies, although this can never overcome the effects of increasing path length (Banavar et al., 1999). In the current framework, this is equivalent to declining $\eta_{S}$ as $X$ and hence $x$ increases. Figure 1 ii suggests $\eta_{S}$ has declined consistently since at least 1900, and if $\mathrm{EROI}_{\mathrm{G}}=\eta / \lambda / k$ at the global scale, then because $\eta_{S}$ is a factor of $\eta$ we should expect $\mathrm{EROl}_{\mathrm{G}}$ to experience similar dynamics as $\eta_{S}$ all else remaining equal.

Jarvis et al., (2015) explored the growth-induced declines in supply efficiency by analysing the scaling relationship between primary and final energy flows. Figure $1 \mathrm{i}$ inset shows this relationship for the De Stercke (2014) data. These data give $x_{A} \propto x^{0.87}$ and, therefore, $\eta_{S} \propto x$ 0.13 , i.e. $\eta_{S}$ declines at $13 \%$ the rate of growth of $x$ or $-0.3 \% \mathrm{yr}^{-1}$ if $r=2.5 \% \mathrm{yr}^{-1}$. This is less than the $-0.6 \% \mathrm{yr}^{-1}$ estimated by Jarvis et al., (2015) using the IEA data for the period 1970 - 2010. This difference is partly, but not exclusively, explained by the fact that the De Stercke (2014) final energy data do not include losses attributed to mining, quarrying, fishing and agriculture, unlike the Jarvis et al., (2015) compilation.

In exploring how variations in supply efficiency have impacted on $\mathrm{EROI}_{\mathrm{G}}$ we are paralleling what Hall et al., (2014) might refer to as 'point-of-use' EROI, because one is attempting to capture the effects of both acquisition and distribution losses on system performance. However, all else does not remain constant, other than the tendency for return rates to be conserved in the long-run and at the global scale. To preserve these return rates on energy use requires $\eta_{U}$ and/or $\eta_{M}$ be increased to offset any systemic declines in $\eta_{S}$. Figure $1 \mathrm{ii}$ suggests most of the declines in $\eta_{S}$ are offset by increases in $\eta_{U}$ given the product $\eta_{U} \eta_{S}$ is relatively stable at $0.23 \pm 0.04$. This suggests $\eta_{M}$ has also been relatively constant throughout the period at the global scale. For this to be the case, the very substantial improvements in energy efficiency in many manufacturing processes that have occurred 
over the last 100 years would have to be offset by declining efficiency in other areas of the infrastructure manufacturing portfolio, presumably associated with the introduction of new infrastructure classes and their associated novel production processes. Assuming $\eta$ is stationary and the long-run growth rate is specifically $2.7 \% \mathrm{yr}^{-1}$ in the De Stercke (2014) data, then $\eta / \lambda=2(2.7) \% \mathrm{yr}^{-1}$. Using the estimates of $\eta_{U} \eta_{S}$ shown in Figure $1 \mathrm{ii}$, equation (5) gives us an estimate of $\eta_{M}$ of $0.24 \pm 0.04$ i.e. industrial society is $\sim 24 \%$ efficient at translating final energy into infrastructure at the global scale. This suggests the variations about this figure presented in Fig. $1 \mathrm{ii}$ are largely the product of small variations in $\eta / \lambda$ and/or the effects of data uncertainties.

A popular narrative in the EROI literature is the notion of an 'energy cliff' where EROI falls to levels unable to support society as currently conceived (Murphy and Hall, 2011). An alternative and possibly better metaphor would be that any limits on efficiency improvements represent a thermodynamic 'wall' for growth. If growth causes systemic declines in $\eta_{S}$ as I have argued, and we currently offset this diminishing return to scale by raising $\eta_{U}$, then it would be naïve to assume $\eta_{U}$ could be raised without limit. As a minimum, $\eta_{U}$ is limited by the Carnot efficiency of the system. Once such a limit was approached the long-run relative return rates of the global economy could not be preserved and growth would tend to become size-determined, as it is in most biological systems (West et al., 2001). It is not apparent how far we are from such limits, especially as it appears possible to raise $\eta_{U}$ substantially through expansion of direct electricity production (wind, photovoltaic and other approaches), and combined heat and power. Furthermore, a global value for $\eta_{M}$ of $24 \%$ suggests substantial scope for raising this further as well. Clearly it would be very helpful to know what the thermodynamic limits of $\eta_{U}$ and $\eta_{M}$ were.

The present issue is how fast industrial society needs to increase $\eta_{U}$ (or $\eta_{M}$ ) in order to offset the declines in $\eta_{S}$ and hence preserve growth. As stated earlier, the De Stercke (2014) data suggest that $\eta_{S} \propto x^{-0.13}$, or supply efficiency is falling at $\sim 0.3 \% \mathrm{yr}^{-1}$ if primary energy is growing at $\sim 2.5 \% \mathrm{yr}^{-1}$. Therefore, in total $\eta_{U}$ must be currently increasing at $\sim 0.3 \% \mathrm{yr}^{-1}$ to compensate for these growth-induced declines in supply efficiency. Clearly this is not particularly quick, which possibly explains why the uptake rate of innovations on energy saving is much slower than the available portfolio of innovations would suggest is possible or desirable (Department for Energy and Climate Change, 2013). It may also explain why it is very difficult to change this rate as policy often wishes to.

\subsection{Infrastructure turnover and historic variations of global energy returns}

Thus far I have largely considered the long run relative growth rate of the real economy as being constant around $2.5 \% \mathrm{yr}^{-1}$. However, as shown in Figure 1iii, the observed relative growth rate appears to have varied substantially and systematically over the past 100 years or more. If both $r=\eta / \lambda-k$ (from equation (4)), and the overall efficiency, $\eta$, does not vary substantially (as discussed in Section 4), then the variations in $r$ shown in Figure 1iii must be largely attributed to variations in the rate of infrastructure decay, $k$. Figure 1 iii shows an estimate of these variations using the non-stationary estimates of $r$ presented in Figure 1iii 
and the expression $k=\eta / \lambda-r$. Here, again I have assumed $\eta / \lambda$ is equal to twice the long-run growth in GPE, or $0.05 \mathrm{yr}^{-1}$.

Figure 1iii is interesting in that it suggests that the observed 'long wave'-like variations in the growth rate in GPE are the product of the rate at which infrastructure is removed from the global portfolio, rather than the rate at which it is added, as is commonly believed (e.g. Grubler et al., 1999). If we assume the ability of the environment to erode infrastructure is somewhat stationary over this $>100$-year interval, then these variations represent nonstationarity in the timeframe over which people decide to collect returns from energy investments through active decommissioning of 'old' infrastructure. These timescales range from as low as $\sim 0.04^{-1}=25$ years in the depths of the recessions that peaked in the 1930's and 1990's, to as much as $\sim 0.01^{-1}=100$ years in the peak of the 1960's boom.

The more familiar narrative for describing long-wave dynamics is constructed around innovation-led nonlinear logistic episodes of growth (Grübler et al., 1999; Manoli et al., 2016). The account of this behaviour I offer in terms of non-stationary rates of decommissioning of infrastructure is not incompatible with this, given that the removal of old technology is a prerequisite to populating the infrastructure portfolio with new technology. However, a nonlinear account of these observations, such as episodic logistics, could be accused of overcomplicating what appears to be an otherwise near-linear dynamic variation in the relative growth rate of GPE. For example, such behaviour could simply arise out of the implicit desire to maintain growth at some level, but with some lag associated with perceiving and responding to any deviations from this ideal.

If $\mathrm{EROI}_{\mathrm{G}}=\eta / \lambda / k$ as proposed here, then the variations in $k$ presented in Figure $1 \mathrm{iii}$ also have important implications for $\mathrm{EROI}_{\mathrm{G}}$. Figure 1iv presents the estimated variations in $\mathrm{EROI}_{\mathrm{G}}$ assuming $\eta / \lambda$ is equal to twice the long-run growth in GPE, and using the estimates for $k$ presented in Figure 1iii. Not surprisingly, $\mathrm{EROI}_{\mathrm{G}}$ is low in the recessions centred in the 1930's and 1990's when infrastructure lifetimes are low, and rises significantly in the 1960's when infrastructure lifetimes are high. This is fully in line with expectation in terms of equation (6) because the lifetime of an energy investment determines the integration time for the total energy returns from that investment. Based on this I would argue that, providing actual infrastructure lifetimes are being used, any observed declines in EROI measures from the 1960's onwards are likely to reflect the effects of declining infrastructure lifetime over this period to some extent. Of course, the real test for this will be whether EROI estimates are seen to increase significantly if the global economy enters another episode of above longrun average growth as suggested could be happening by the GPE data in Figure 1.

\subsection{Summary and conclusion}

Because of the need to specify system boundaries EROI will remain a conceptually appealing yet difficult to specify parameter. This is particularly true when attempting to accommodate the effects of infrastructure investments and how returns from these accrue over time. Here I have argued that matters could be greatly simplified when analysing a largely undifferentiated global scale system because there are far fewer boundaries to consider. In doing so, although you sacrifice the ability to differentiate between sub- 
components of this system, you are able to construct a much clearer picture of the global scale drivers for growth.

The only differentiation I offered was to distinguish between the useful, supply and infrastructure manufacturing components of GPE, and by implication, the fraction of these components that are wasted. I attempted to show that a central feature of these components was a declining return-to-scale associated with growth-induced decreases in resource supply efficiency. However, the observed long-run exponential character of GPE suggests a strong tendency for the global economy to self-organise such that, overall, energy efficiency is preserved, and hence long-run net returns remain close to $2.5 \% \mathrm{yr}^{-1}$. I attempted to show this was associated with an $\mathrm{EROI}_{\mathrm{G}}$ of $2: 1$, realised with a time constant of 40 years and a break-even time of 28 years.

The De Stercke (2014) data used here also reveal significant 'wave-like' variation in the relative growth rate of GPE about $\sim 2.5 \% \mathrm{yr}^{-1}$. The apparent preservation of the overall efficiency of energy use implicates variations in the decommissioning/decay rate of infrastructure in these dynamics. This view is not incompatible with current thinking on innovation dynamics in the global economy, but points to a simple explanation focused on lags in any attempt to correct for deviations from $\sim 2.5 \% \mathrm{yr}^{-1}$. This view is one where a certain level of growth is the economic objective, something that fits with the prevailing narrative around avoiding recessionary and inflationary episodes. In the absence of an obvious single controlling hand at the global scale, and in light of the heterogeneity in the global economy, this suggests such behaviour is truly emergent.

The estimates of usefulness and manufacturing efficiencies suggest significant headroom is available for accommodating further declines in supply efficiency in order to preserve growth. Therefore, although we appear to be seeing the signs of declines in the growth opportunities of certain highly developed economies (e.g. Japan, Western Europe), it would also appear this is not the case globally and emerging economies like China and India look set to further consume this headroom. Indeed, if the GPE data are to be believed, real growth looks set to move above its long run value for the next two decades or more. However, at some point the system must hit its thermodynamic limits where there is no residual energy flow to invest in additional infrastructure, and hence $\mathrm{EROI}_{\mathrm{G}}=1$. The enhanced decay of infrastructure elicited by anthropogenic climate change should be a more pressing issue for those concerned about such things.

\section{Acknowledgements}

This paper benefitted significantly from the thoughtful and detailed review comments of Carey King and Charles Hall. 


\section{References}

Ayres, R.U. (1989). U.S. Competitiveness in Manufacturing. IIASA Research Report. IIASA, Laxenburg, Austria: RR-89-006.

Ayres, R. Nair, I (1984). Thermodynamics and economics. Physics Today, 25: 62-71.

Ayres RU, Warr B (2010). The Economic Growth Engine: How Energy and Work Drive Material Prosperity.

Banavar JR, Maritan A, Rinaldo A (1999). Size and form in efficient transportation networks. Nature 399, 130-132.

Bhattacharyya SC, Timilsina GR, (2010). A review of energy system models. International Journal of Energy Sector Management, 4, 494-518.

Brandt AR, Dale M, Barnhart CJ. (2013) Calculating systems-scale energy efficiency and net energy returns: a bottom-up matrix-based approach. Energy, 62 (0): 235e47.

British Petroleum Statistical Review of World Energy (2011)

http://www.bp.com/assets/bp_internet/globalbp/globalbp_uk_english/reports_and_publicatio ns/statistical_energy_review_2011/STAGING/local_assets/spreadsheets/statistical_review_ of_world_energy_full_report_2011.xls. (accessed - 10/06/2011)

Cleveland CJ, Costanza R, Hall CAS, Kaufmann R (1984). Energy and the U.S. Economy: A Biophysical Perspective. Science, 225, 890-897.

Davis SJ, Caldeira K., Matthews DH. (2010) Future $\mathrm{CO}_{2}$ emissions and climate change from existing energy infrastructure, Science, 329, 1330-1333.

Dale M, Krumdieck S, Bodger P (2012a) Global energy modelling - A biophysical approach (GEMBA) Part 1: An overview of biophysical economics. Ecological Economics, 73, 152157.

Dale M, Krumdieck S, Bodger P (2012b) Global energy modelling - A biophysical approach (GEMBA) Part 2: Methodology. Ecological Economics, 73, 158-167.

Department for Energy and Climate Change (2013). Statistical Release. Experimental Statistics Department of Energy and Climate Change (DECC), London, UK (2013)

Dennison EF (1979) Explanation of declining productivity growth. Survey of Current Business, 59/8, Part II, 1-24

De Stercke S (2014). The Primary, Final and Useful Energy Database (PFUDB). http://www.iiasa.ac.at/web/home/research/researchPrograms/TransitionstoNewTechnologies /PFUDB.en.html (accessed 02/11/2016) 
Garrett TJ (2011) Are there basic physical constraints on future anthropogenic emissions of carbon dioxide? Climatic Change, 104, 437

Garrett, TJ (2012). "No way out? The double-bind in seeking global prosperity alongside mitigated climate change. Earth System Dynamics, 3, 1-17.

Garrett, (2015) Long-run evolution of the global economy - Part 2: Hindcasts of innovation and growth. Earth System Dynamics, 6, 673-688.

Grübler A (2008). Technology and Global Change (Cambridge University Press) (associated world primary energy use data downloaded from:

http://user.iiasa.ac.at/ gruebler/Data/TechnologyAndGlobalChange/w-energy.csv)

Grübler A, Nakienovic N, Victor GB (1999) Dynamics of energy technologies and global change, Energy Policy, 27, 247-280

Grubler A, Johansson TB, Mundaca L, Nakicenovic N, Pachauri S, Riahi K, Rogner H-H, Strupeit L (2012): Chapter 1 - Energy Primer. In Global Energy Assessment - Toward a Sustainable Future, Cambridge University Press, Cambridge, UK and New York, NY, USA and the International Institute for Applied Systems Analysis, Laxenburg, Austria, pp. 99-150.

Guilford MC, Hall CAS, O' Connor P, Cleveland CJ (2011) A New Long Term Assessment of Energy Return on Investment (EROI) for U.S. Oil and Gas Discovery and Production. Sustainability, 3, 1866-1887.

Hall CAS, Cleveland CJ, Kaufman R (1986). Energy and Resource Quality: The Ecology of the Economic Process: John Wiley \& Sons.

Hall CAS, Lambert JG, Balogh SB (2014). EROI of different fuels and the implications for society. Energy Policy, 64, 141-152.

Hendrickson C, Horvath A, Joshi S, Lave L (1998). Economic Input-Output Models for Environmental Life-Cycle Assessment. Environmental Science and Technology, 1, 184-191.

Herendeen RA (2015) Connecting net energy with the price of energy and other goods and services. Ecological Economics, 109, 142-149

Jarvis AJ, Leedal DT, Hewitt CN (2012). Climate-society feedbacks and the avoidance of dangerous climate change. Nature Climate Change, 2, 668-671.

Jarvis AJ, Hewitt CN (2014) The "Business-As-Usual" growth of global primary energy use and carbon dioxide emissions - historical trends and near-term forecasts. Earth System Dynamics Discussions, 5, 1143.

Jarvis AJ, Jarvis SJ and Hewitt CN (2015) Resource acquisition, distribution and end-use efficiencies and the growth of industrial society. Earth System Dynamics, 6, 1-14. 
Jebaraj S, Iniyan S (2006). A review of energy models. Renewable \& Sustainable Energy Reviews, 10, 281-311

King CW (2014) Matrix method for comparing system and individual energy return ratios when considering an energy transition. Energy, 72, 254-265.

Kummel R (2011) The second law of economics: Energy, Entropy, and the Origins of Wealth. Springer.

Lenzen M, (2001) Errors in conventional and input-output-based life cycle inventories. J. Industrial Ecology, 4, 127-148.

Louwen A, van Sark WGJHM, Faaij APC, Schropp REI (2016) Re-assessment of net energy production and greenhouse gas emissions avoidance after 40 years of photovoltaics development. Nature Communications, 7, 13728

Manoli, G, Katul GG, Marani M (2016), Delay-induced rebounds in CO2 emissions and critical time-scales to meet global warming targets, Earth's Future, 4, 636-643.

Murphey DJ, Hall CAS (2011) Energy return on investment, peak oil, and the end of economic growth. Ecological Economics Reviews, 1219, 52-72

Rodrigue J-P, Comtois C, Slack B (2009) The "Last Mile" in Freight Distribution. The Geography of Transport Systems (2nd ed.). Routledge.

Stern DI (2010) The Role of Energy in Economic Growth. CCEP working paper 3.10, Centre for Climate Economics \& Policy, Crawford School of Economics and Government, The Australian National University, Canberra

West GB, Brown JH, Enquist BJ, (2001) A general model for ontogenetic growth. Nature, $413,628-631$. 
Table 1. Simple linear regressions results for the data shown in Figure 1i. All parameter uncertainties are reported to one standard deviation.

\begin{tabular}{|c|c|c|}
\hline Model & Parameter estimates & $r^{2}$ \\
\hline $\ln (\mathrm{GPE})=a\left(t-t_{1}\right)$ & $\begin{aligned} a & =0.027 \pm 0.004 \\
t_{1} & =1775 \pm 2.7 \mathrm{AD}\end{aligned}$ & 0.979 \\
\hline $\ln (\mathrm{GWP})=a\left(t-t_{1}\right)$ & $\begin{aligned} a & =0.031 \pm 0.004 \\
t_{1} & =1887 \pm 1.1 \mathrm{AD}\end{aligned}$ & 0.977 \\
\hline $\ln (\mathrm{GFE})=a \ln (\mathrm{GPE})+c$ & $\begin{array}{l}a=0.8733 \pm 0.0045 \\
c=0.1289 \pm 0.0224\end{array}$ & 0.997 \\
\hline $\ln (\mathrm{GUE})=a \ln (\mathrm{GPE})+c$ & $\begin{array}{l}a=1.2115 \pm 0.0056 \\
c=-2.3066 \pm 0.0281\end{array}$ & 0.998 \\
\hline $\ln (\mathrm{GWP})=a \ln (\mathrm{GPE})+c$ & $\begin{array}{c}a=1.1378 \pm 0.010 \\
c=-3.4076 \pm 0.0523\end{array}$ & 0.991 \\
\hline
\end{tabular}



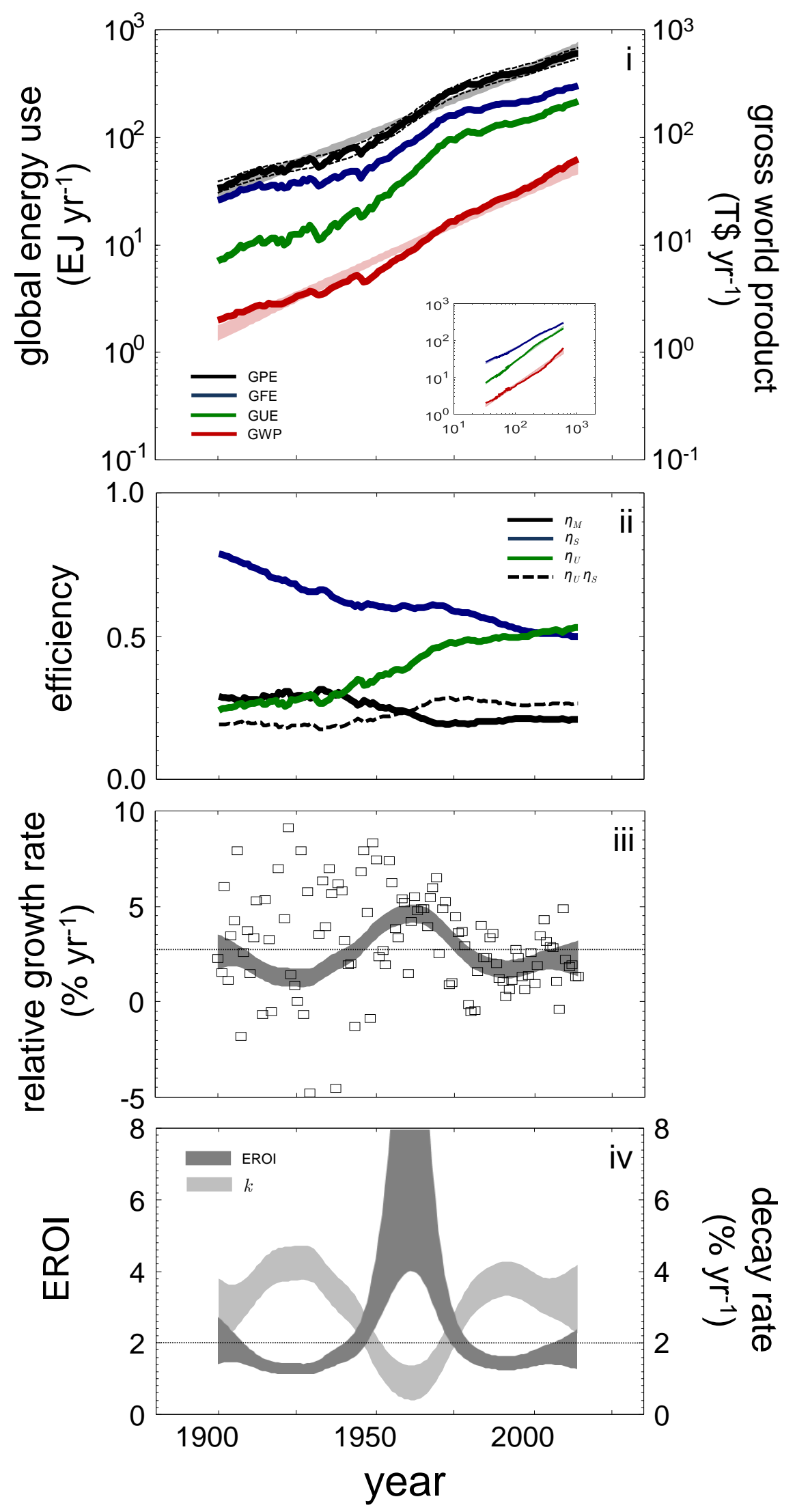
Fig. 1 i. Global Primary Energy (GPE), Global Final Energy (GFE), Global Useful Energy (GUE) and Gross World Product (GWP), 1900 to 2014. All data are from De Stercke (2014), although total transport has also been subtracted from the GFE series (see text for explanation). The shaded areas are $95 \%$ confidence intervals from the simple linear regression results reported in Table 1. The dashed lines are 95\% confidence intervals associated with the smoothed GPE relative growth rates presented in iii. The inset shows the scaling relationships between GPE and GFE, GUE and GWP, with shaded areas again representing $95 \%$ confidence intervals for the simple linear regression results reported in Table 1 . ii. The supply efficiency, $\eta_{S}$, usefulness efficiency, $\eta_{U}$, and manufacturing efficiency, $\eta_{M}$, estimated from the data in i. (see text for explanation). Also shown is the product $\eta_{U} \eta_{S}$. iii. The relative growth rate, $r$, of the GPE data shown in i. Also shown is the $95 \%$ confidence interval of a fixed interval smoothed estimate of $r$, again made from the GPE data in i. The stationary estimate of $2.7 \% \mathrm{yr}^{-1}$ from the simple linear regression of these same data is shown as the dashed line for reference. iv. $95 \%$ confidence interval for the non-stationary estimates of both the global aggregate decay rate of infrastructure, $k$, and $\mathrm{EROI}_{\mathrm{G}}$ (see text for explanation). 


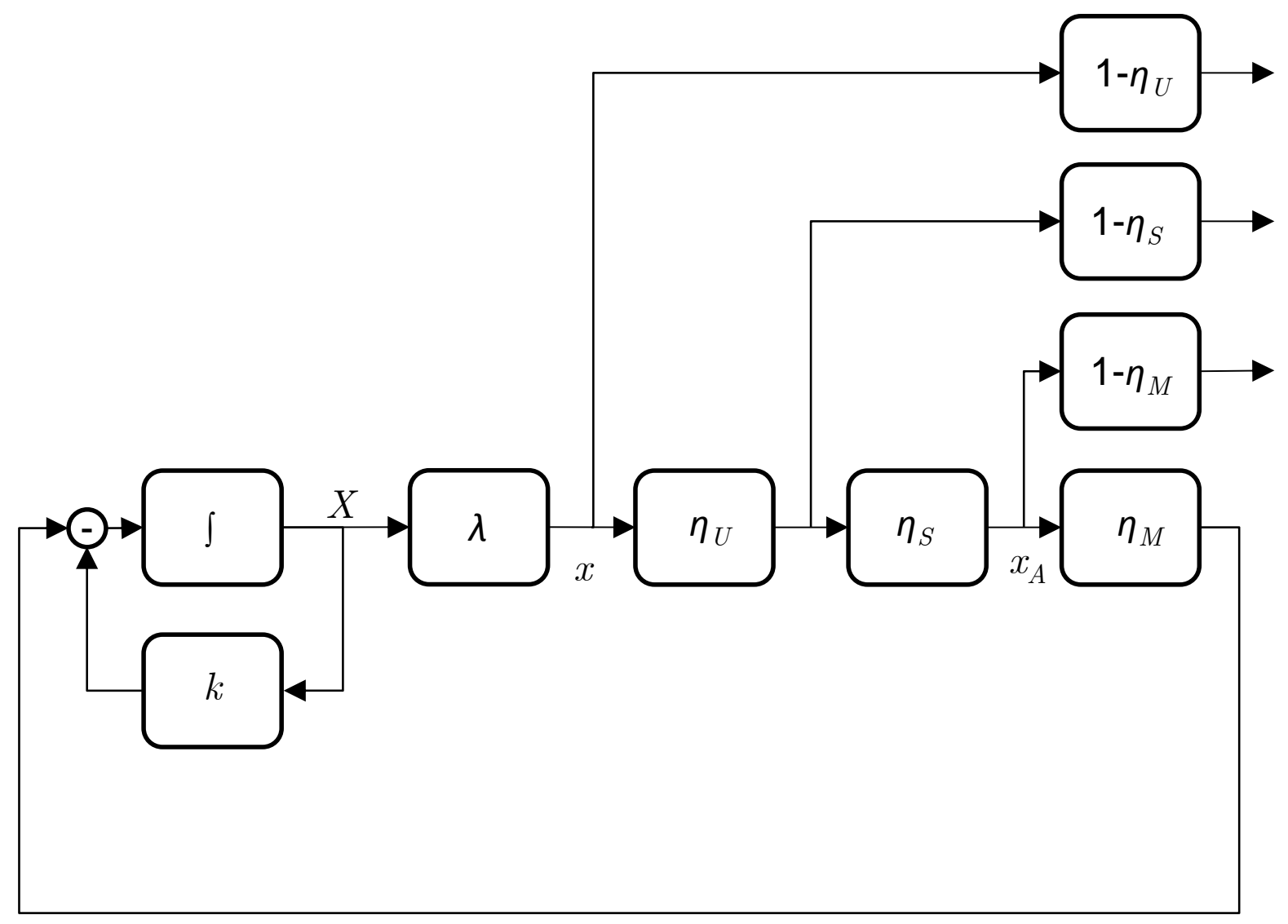

Figure 2. System block diagram of the proposed endogenous growth model structure. 


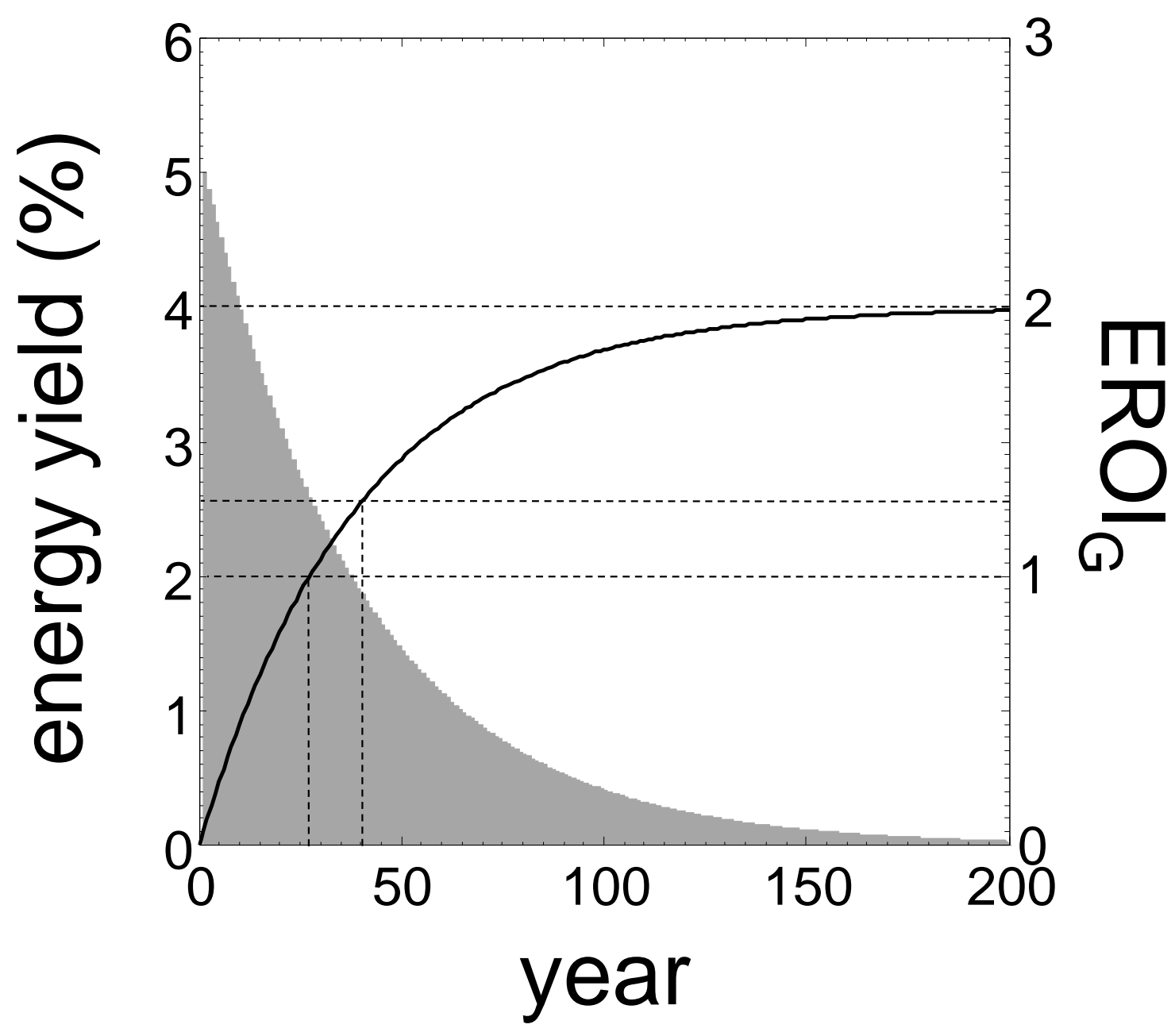

Figure 3. The impulse response of equation (8) showing how global energy outputs decay following a unit input of primary energy into the global economy (grey shaded area). Also shown is the cumulative energy output which, for the unit input case, represents the time evolution of $\mathrm{EROI}_{\mathrm{G}}(-)$ again following a unit input of primary energy. Also shown is the half time and time constant of the $\mathrm{EROI}_{\mathrm{G}}$ response where the impulse response has decayed by 50 and $63 \%$ respectively (vertical dashed lines). 